\title{
Expression of coxsackie and adenovirus receptor distinguishes transitional cancer states in therapy-induced cellular senescence
}

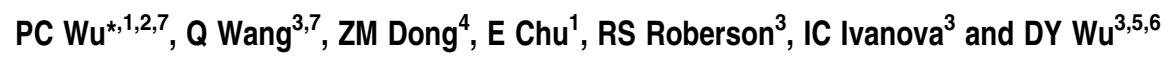

\begin{abstract}
Therapy-induced cellular senescence describes the phenomenon of cell cycle arrest that can be invoked in cancer cells in response to chemotherapy. Sustained proliferative arrest is often overcome as a contingent of senescent tumor cells can bypass this cell cycle restriction. The mechanism regulating cell cycle re-entry of senescent cancer cells remains poorly understood. This is the first report of the isolation and characterization of two distinct transitional states in chemotherapy-induced senescent cells that share indistinguishable morphological senescence phenotypes and are functionally classified by their ability to escape cell cycle arrest. It has been observed that cell surface expression of coxsackie and adenovirus receptor (CAR) is downregulated in cancer cells treated with chemotherapy. We show the novel use of surface CAR expression and adenoviral transduction to differentiate senescent states and also show in vivo evidence of CAR downregulation in colorectal cancer patients treated with neoadjuvant chemoradiation. This study suggests that CAR is a candidate biomarker for senescence response to antitumor therapy, and CAR expression can be used to distinguish transitional states in early senescence to study fundamental regulatory events in therapy-induced senescence.
\end{abstract}

Cell Death and Disease (2010) 1, e70; doi:10.1038/cddis.2010.47; published online 2 September 2010

Subject Category: Cancer

Cellular senescence, elicited physiologically by telomere attrition, can also be triggered by DNA damage and selected oncogenic signals. ${ }^{1-4}$ In pre-cancerous states such as melanocytic nevus or dysplastic Barrett's esophagus, cellular senescence has been postulated to enforce growth arrest and functionally suppress tumor transformation. ${ }^{5-8}$ Activation of tumor suppressor pathways mediated by p53 and pRB is typically required for the induction and maintenance of the senescent response..$^{9-11}$ Unfortunately, neoplastic cells can bypass cell cycle restriction through mutational, epigenetic or functional inactivation of p53 and pRB pathways during carcinogenesis to resume proliferation. ${ }^{9,10,12}$ For example, nonrandom gains of chromosomes $5 q, 8 q$ and 10 in prostate epithelial cells have been shown to have a key role in senescence bypass (or escape) in the pathogenesis of prostate cancer. $^{12}$

Therapy-induced cellular senescence has been shown in a variety of in vitro and in vivo models and in human tumor samples. ${ }^{1,13-16}$ Despite the inactivation of p53 and p16 ${ }^{\text {INK4a/ }}$ pRB pathways in the majority of human cancer types, chemotherapeutic drugs and ionizing radiation can elicit robust senescence responses in many tumor cell lines. ${ }^{1,11,17,18}$ Senescent cells are characterized by sustained proliferative arrest, morphological changes and expression of the classical senescence marker, $\mathrm{pH}$-restricted, senescenceassociated $\beta$-galactosidase (SA- $\beta$-gal). Escape from therapyinduced senescence has been shown, but the mechanism regulating cell cycle re-entry of senescent cell remains poorly understood. In chemotherapy-treated senescent p53-null and p16-deficient $\mathrm{NCl}-\mathrm{H} 1299$ cells, we have shown that senescence escape is closely associated with the aberrant over-expression of the cyclin-dependent kinase Cdc2/Cdk1. ${ }^{19}$ In an effort to gain a better understanding of this biological barrier, we sought to further characterize early events during senescence escape. This is the first descriptive report of the isolation and characterization of two distinct transitional states in chemotherapy-induced senescence distinguished by the ability of senescent cells to escape cell cycle arrest. Despite sharing indistinguishable morphological senescence phenotypes, the two states can be differentiated by their surface expression of Coxsackie and adenovirus (Adv) receptor (CAR).

$\mathrm{CAR}$ is a $46-\mathrm{kDa}$ glycosylated transmembrane protein associated with tight junction complexes and appears to have important roles in embryonic development, cell-cell adhesion and intercellular recognition. ${ }^{20,21}$ Cell surface CAR expression enables viral attachment by its interaction with the Adv finger-knob protein and functions to regulate viral uptake,

\footnotetext{
${ }^{1}$ Department of Surgery, VA Puget Sound Health Care System, Seattle, WA, USA; ${ }^{2}$ Department of Surgery, University of Washington, Seattle, WA, USA; ${ }^{3}$ Department of Medicine, VA Puget Sound Health Care System, Seattle, WA, USA; ${ }^{4}$ Department of Pathology, VA Puget Sound Health Care System, Seattle, WA, USA; ${ }^{5}$ Department of Medicine, Division of Oncology, University of Washington, Seattle, WA, USA and ${ }^{6}$ Fred Hutchinson Cancer Research Center, Seattle, WA, USA

${ }^{*}$ Corresponding author: PC Wu, Department of Surgery, University of Washington, 1660 S. Columbian Way (S-112), Seattle, WA 98108, USA. Tel: 2 06 768 5287; Fax: 206764 2529; E-mail: pcwu@u.washington.edu

${ }^{7}$ These authors contributed equally to this work.

Keywords: cellular senescence; coxsackie and adenovirus receptor; transition state; biomarker

Abbreviations: Adv, adenovirus; CAR, coxsackie and adenovirus receptor; CK20, cytokeratin 20; CPT, camptothecin; GFP, green fluorescence protein; mut, mutant; RFP, red fluorescence protein; SA- $\beta$-gal, senescence-associated $\beta$-galactosidase

Received 19.5.10; revised 21.7.10; accepted 26.7.10; Edited by A Finazzi-Agro'
} 
cytoskeleton and cell migration. ${ }^{22,23}$ Recently, CAR knockdown in a colon cancer line has been shown to strongly downregulate $\alpha$-catenin and alter cellular morphology, increase cell proliferation and enhance cell migration and invasion. ${ }^{24}$ CAR is ubiquitously expressed in many normal epithelial tissues, but its expression appears to vary in tumor tissues with advanced grade, stage and increased metastatic potential. $^{24,25}$

In this report, we show that surface CAR downregulation is a consistent feature observed in multiple cell lines during chemotherapy-induced senescence. Using either surface CAR expression or a marker Adv transduction as a surrogate determinant for CAR, senescent cell populations can be identified and separated into two morphologically indistinguishable subpopulations that functionally differ by their ability to escape senescence. The subpopulation of senescent cells, characterized by high surface CAR expression, retains the ability to escape cell cycle arrest. Conversely, low CAR-expressing senescent cells are confined to a prolonged senescent state and destined for eventual cell death. The biological events underlying the transition between these two states constitute a significant biological barrier that determines the reversibility of therapy-induced senescence and cell fate. We also provide evidence of downregulation of surface CAR in human rectal cancer specimens following neoadjuvant (preoperative) chemotherapy and radiation. Altogether, our work proposes CAR as a novel biomarker for senescence in response to antitumor therapy, and that surface CAR expression may be a means for isolating distinct transitional states within therapy-induced senescence whose biological differences may yield novel insights into barriers of senescence.

\section{Results}

Chemotherapy-induced senescent cells downregulate surface expression of CAR. Previous studies have shown that cells in replicative senescence may become less susceptible to adenoviral infection as a result of surface CAR downregulation. ${ }^{26,27}$ We therefore sought to determine whether CAR is similarly downregulated in therapy-induced senescent cells in an established tissue culture system. ${ }^{19}$ In the schema depicted in Figure 1a, immortalized cancer cells adopt a state of prolonged arrest and exhibit classical senescence phenotype within 2-6 days following an abbreviated exposure to moderate concentrations of chemotherapy. For example, $30 \%$ of $\mathrm{H} 1299$ cells treated with camptothecin (CPT) at $0.3-2 \times \mathrm{IC}_{50}$ for 3 days remain viable upon release into fresh media. Among these cells, $>70-80 \%$ become morphologically senescent and expresses the senescence marker SA- $\beta$-gal. These senescent cells persist for up to 3 weeks, during which infrequent cells escape cell cycle arrest to form proliferative colonies. To determine whether surface CAR is downregulated during therapyinduced senescence, we performed immunofluorescence staining with anti-CAR antibody in unfixed, non-permeabilized cells. Three independent tumor lines including $H 1299$ (lung), $H C T 116$ (colon) and $B x P C-3$ (pancreas) were treated with either CPT or doxorubicin, recovered and examined by
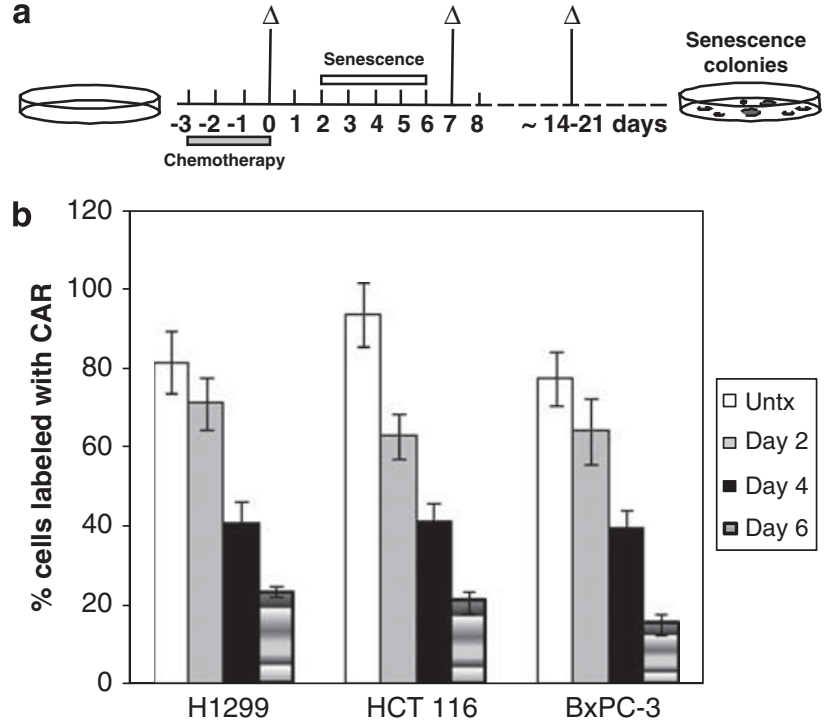

Figure 1 Surface CAR expression in senescent H1299, HCT 116 and BxPC-3 cells. (a) Therapy-induced senescence in vitro schema: tumor cells are treated for 3 days ( -3 to 0 ) with CPT ( $30 \mathrm{nM}$ for H1299; $120 \mathrm{nM}$ for BxPC-3) or doxorubicin (150 nM for HCT 116) and rinsed on day 0 . Fresh media replaced $(\Delta)$ every 7 days until the formation of colonies within 14-21 days. Classical senescence morphology is observed between days 2 and 6. (b) Quantitative histogram of cell surface CAR expression by immunostaining with anti-CAR antibody and FACS analysis. Untx represents untreated cells. Error bars denote standard deviation of three independent experiments $(N=3)$

flow-activated cell sorting (FACS) analysis (Figure $1 \mathrm{~b}$ and Supplementary Figure 1A). All three cell lines exhibited consistent and significant $(>75 \%)$ reduction of their surface CAR expression over a period of 6 days in senescence. The reduction of surface CAR expression appears to be the result of membrane protein downregulation as the total cellular CAR level is found to be only modestly reduced over this same period of time (Supplementary Figures $1 \mathrm{~B}$ and $\mathrm{C}$ ).

Therapy-induced senescent cells lose susceptibility to adenoviral infection as a result of surface CAR downregulation. CAR expression has been shown to correlate with the efficiency of adenoviral-mediated transduction in a variety of human malignancies, including glioma, melanoma, lung and pancreatic cancer. ${ }^{28-31}$ Following their release from chemotherapy exposure, we have observed that senescent cells become progressively resistant to adenoviral infection. As shown in Figure $2 \mathrm{a}$, significant numbers of $\mathrm{H} 1299$ tumor cells on day 4 fail to express the marker red fluorescence protein (RFP) after a $4 \mathrm{~h}$ infection with Adv harboring the RFP transgene (Adv-RFP). In contrast, nearly all untreated cells express RFP after identical Adv-RFP infection. Quantitative FACS analysis further shows that senescent cells of all three tumor lines become progressively resistant to Adv-RFP marking (Figure $2 \mathrm{~b}$ and Supplementary Figure $2 \mathrm{~A}$ ). In each case, the percentage of RFP-expressing cells following marker virus infection declines by at least $75 \%$ over 6 days. Using the uninfected senescent tumor cells to set FACS gating, Adv-RFP-infected cells can be differentiated as either 'marked' cells (i.e., RFP expression above the uninfected 

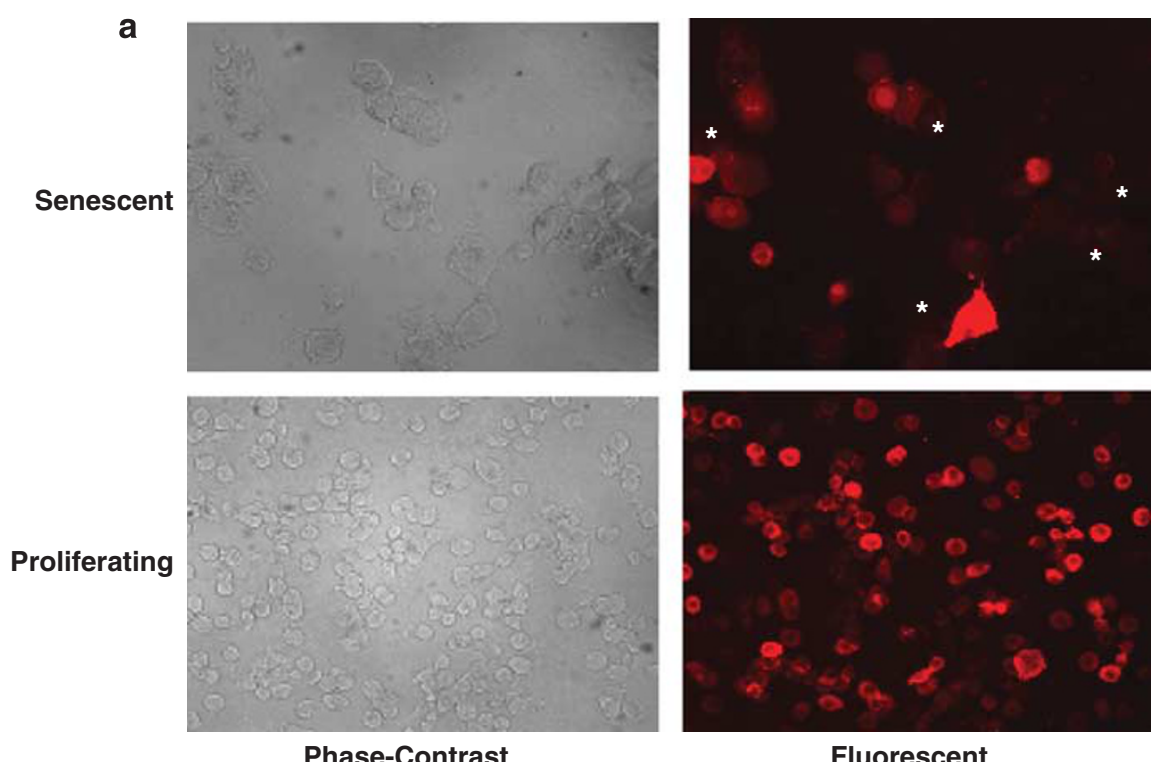

Phase-Contrast

Fluorescent

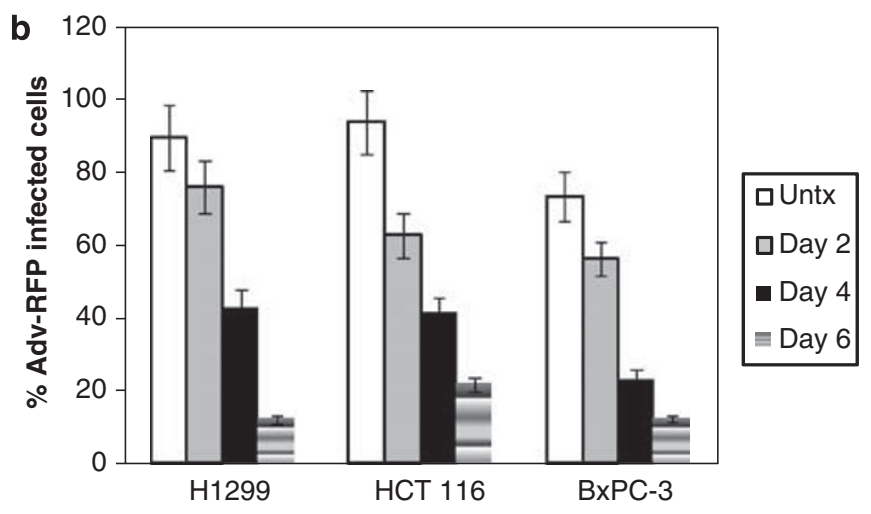

Figure 2 Susceptibility of therapy-induced senescent H1299, HCT 116 and BxPC-3 cells to Adv-RFP infection: H1299, BxPC-3, and HCT 116 cells were treated as described in Figure 1 and infected with Adv-RFP at indicated days and resultant RFP expression determined by FACS analysis. (a) Phase-contrast and fluorescence microscopy images $(\times 40)$ of proliferating and senescent (day 4) H1299 cells. Asterisks indicate 'unmarked' senescent cells lacking RFP expression. (b) Histogram of gated results of 'marked' RFP-transduced senescent cells. Data points are shown as mean with error bars representing standard deviation of three independent experiments $(N=3)$. Asterisks $\left(^{*}\right)$ indicate 'unmarked' senescent cells lacking RFP expression

baseline) or as 'unmarked' cells (i.e., RFP expression at or below the baseline). The marked and unmarked senescent cells are otherwise indistinguishable in their morphological features and SA- $\beta$-gal expression (Supplementary Figure 2B).

We next determined a correlation between surface CAR expression and adenoviral susceptibility through a regression analysis using Adv-RFP marking data derived from all three tumor lines and their surface CAR expression (Figure 3a). We found that adenoviral marking correlated closely with CAR expression with a linear correlation coefficient $\left(r^{2}\right)$ of 0.891 and $P$-value of 0.00054 , thereby supporting a linear relationship of these events. To further study this correlation, senescent $\mathrm{H} 1299$ cells harvested 4 days following CPT treatment were sorted into low and high CAR-expressing populations (Figure 3b). The two sorted cell populations were then recovered in fresh media, infected with the marker AdvRFP and analyzed for RFP expression. The results showed threefold greater Adv-RFP marking (67\%) in the high CARexpressing cells as compared with only $22 \%$ marking in the
Iow CAR-expressing cells. Moreover, the anti-CAR antibody in logarithmically increasing concentrations can block adenoviral marking of $\mathrm{H} 1299$ cells (Figure 3c). Linear regression analysis of this experiment resulted in an $r^{2}$ coefficient of 0.99 and $y$-intercept of 0.057 . Altogether, these findings confirm CAR to be the dominant receptor that determines Adv entry into tumor cells and validate the use of adenoviral marking as a surrogate for surface CAR expression in therapy-induced senescent cells.

CAR expression and adenoviral marking is cell cycle independent. It is feasible that the gradual loss of CAR expression and adenoviral marking in therapy-induced senescent cells may be explained by the resistance of senescent cells arrested at G2/M to Adv infection. To determine whether CAR expression and adenoviral marking correlate with cell cycle, we performed a gated analysis of adenoviral marking and CAR expression (Figure 4a). Untreated proliferating $\mathrm{H} 1299$ cells were either immunostained with anti-CAR antibody or marked with Adv harboring 


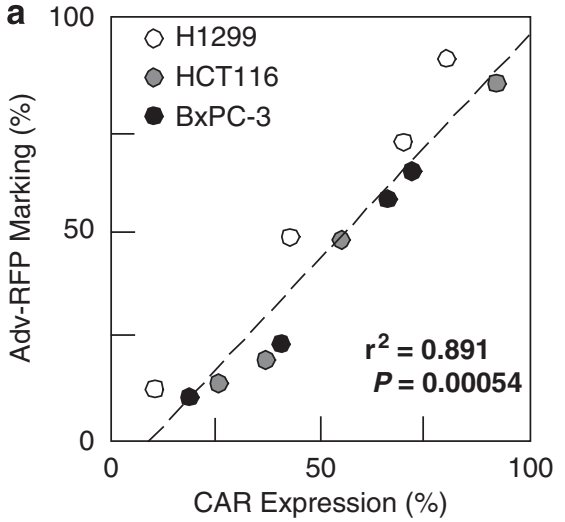

b
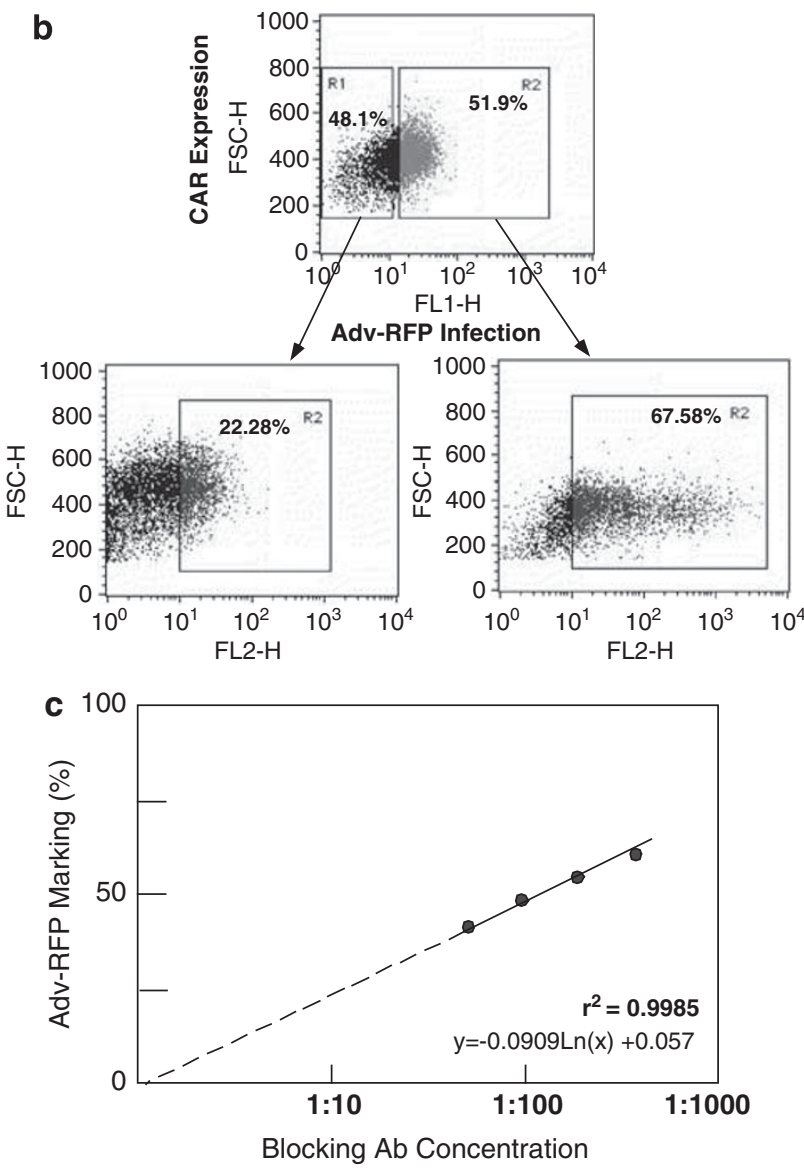

Figure 3 Relationship of adenoviral marking and CAR expression. (a) Linear regression analysis of CAR expression and Adv-RFP infectivity of H1299, HCT 116 and BxPC-3 tumor cells following chemotherapy treatment. (b) Marker virus susceptibility of sorted senescent H1299 cell-based surface CAR expression. Sorted cells $\left(1 \times 10^{5}\right)$ with either low (R1) or high CAR (R2) expression were re-infected with Adv-RFP and resultant RFP expression measured by FACS and gated results reported. (c) Adenoviral marking of $\mathrm{H} 1299$ cells examined in the presence of blocking anti-CAR antibody. Linear regression analysis of Adv-RFP marking fraction and logarithmic antibody concentrations is shown. Each of these experiments has been performed in duplicate $(N=2)$

the green fluorescence protein transgene (Adv-GFP) before fixation and staining with propidium iodide. These cells were gated by FACS to determine either the Adv-GFP marking or the CAR expression in ungated (total), G1 and G2/M fractions. We found that nearly $100 \%$ of $\mathrm{G} 1$ and $\mathrm{G} 2 / \mathrm{M}$ cells express GFP and CAR. We also induced cell cycle arrest at $\mathrm{G} 1$ by serum starvation for $72 \mathrm{~h}$, which resulted in a predominant but incomplete G1 arrest and at G2/M with $20 \mathrm{~nm}$ nocodazole (Figure 4b). Untreated and arrested cells were then infected with Adv-GFP or immunostained for CAR. As shown in Figure 4b, between 89 and $100 \%$ of arrested cells expressed CAR and were marked by Adv-GFP. These results show that features of CAR expression and adenoviral susceptibility are cell cycle independent and represent a biological property of therapy-induced cellular senescence.

Escape from replicative arrest preferentially occurs in the marked senescent cells. In addition to their indistinguishable morphological features, marked and unmarked senescent cells retain their membrane integrity and cellular viability (Supplementary Figures 2B and 3). To determine whether escape occurs preferentially from either the marked or the unmarked senescent cell populations, we sorted Adv-RFP marked, senescent H1299 cells at indicated time points following CPT exposure (Figure 5a). On day 4, FACS sorting efficiently separate marked and unmarked senescent cells based on gates predefined with corresponding uninfected senescent control (Figure 5a, top panel). Equal numbers $\left(5 \times 10^{4}\right)$ of sorted cells from marked and unmarked subpopulations from days 2, 4 and 6 were released into fresh media and allowed to form colonies for an additional 14-21 days. Substantially greater numbers of escape colonies were observed to form from the marked cell population (table in Figure 5a). In contrast, between 10- and 150-fold fewer colonies formed from the unmarked sorted cells. A representative example of a day 4 colony formation assay is shown (picture in Figure 5a). FACS sorting was noted to become less efficient on day 6 , likely attributable to greater pleomorphism and cellular complexity of senescent cells with time. We speculate that the small but significant increase in colony formation from day 6 unmarked cells is the result of declined sorting efficiency. As a complimentary approach, we examined colonies derived from the senescent H1299 cells infected with marker Adv harboring the bacterial LacZ transgene on days 4 and 6 . In situ $\beta$-galactosidase $(\mathrm{pH} 7.0)$ assay was carried out to determine marker protein expression during early colony formation (Figure $5 \mathrm{~b}$ and Supplementary Figure 4A). We reasoned that if colonies can form equally from both senescent states as differentiated by viral marking, then the frequency of detectable colonies (Figure 5b 'expected') should mirror the proportion of marked cells shown in Figure $2 b$, which steadily decreases from days 0 to 6 . However, we found that every colony examined (25/25) at each time point exhibits detectable marker expression with an overall frequency of $100 \%$ (Figure $5 b$ 'results'). This finding, in combination with the data obtained from the colony formation assay in Figure $5 \mathrm{a}$, suggests that escape colonies are derived exclusively from the marked cell subpopulation. CAR expression and adenoviral marking consequently define two functionally different subpopulations of senescent cells with opposing cell cycle re-entry potential and cell fates.

The conversion from a 'marked' to an 'unmarked' state occurs during senescence. The dramatic differences in 


\begin{tabular}{|l|c|c|c|}
\cline { 2 - 4 } \multicolumn{1}{|c|}{} & \multicolumn{3}{|c|}{ Gating } \\
\hline & Ungated & G1 & G2/M \\
\hline Adv-GFP Mark & $100 \%$ & $100 \%$ & $100 \%$ \\
\hline CAR Expression & $99.5 \%$ & $99.3 \%$ & $97.9 \%$ \\
\hline
\end{tabular}
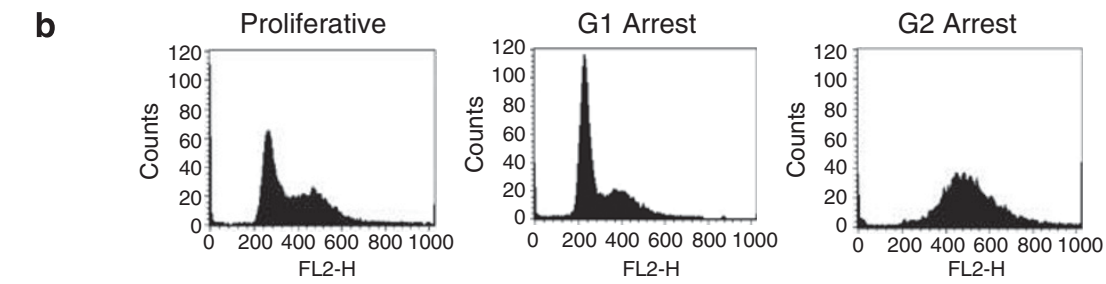

\begin{tabular}{|c|c|c|c|c|c|c|}
\cline { 2 - 6 } & Cont. & Mark/Ab & Cont. & Mark/Ab & Cont. & Mark/Ab \\
\cline { 2 - 7 } & $1.8 \%$ & $100 \%$ & $1.6 \%$ & $99.8 \%$ & $0.5 \%$ & $99.1 \%$ \\
\cline { 2 - 7 } CAR Expression & $2.8 \%$ & $96.8 \%$ & $2.8 \%$ & $96.2 \%$ & $2.1 \%$ & $89.6 \%$ \\
\hline
\end{tabular}

Figure 4 Cell cycle correlation of CAR expression and Adv marking. (a) Proliferative H1299 cells were marked with Adv-GFP or stained with CAR before fixation and stained with propidium iodide. FACS gates were set to determine adenoviral marking or CAR expression of total (ungated), G1 and G2/M cell fractions. (b) H1299 cells were serum starved for 3 days to induce G1 arrest or treated with $20 \mathrm{~nm}$ nocodazole to induce G2 arrest. Proliferating and arrested cells were marked with Adv-GFP or stained with CAR. Percentage of adenoviral marked and CAR-expressing cells were determined by FACS. Control (Cont.) denotes either blank adenoviral infection or staining without antibody. Standard deviation was found to be less than $2 \%$ for all experiments $(N=3)$

escape colony formation between marked and unmarked states, and the progressive decline of adenoviral susceptibility in senescent cells raise the possibility that cells arrested initially in a marked state are steadily converted to an unmarked state. To examine this hypothesis, we performed a tandem adenoviral marking experiment (Figure 5c) where untreated and treated $\mathrm{H} 1299$ tumor cells were initially marked with Adv-RFP and re-infected with a second marker virus, Adv-GFP, at a subsequent time. The re-infection efficiency was determined as the percentage of dual RFP/GFP-expressing cells within the gated RFPexpressing cells. As shown in Figure 5c, untreated H1299 cells can be re-infected with Adv on successive days without significant loss of viral infectivity. The efficiency of the second infection remained $>90 \%$ at 6 days following the primary infection. When the second infection with Adv-GFP was carried out on CPT-treated senescent cells, the efficiency of the second viral marking declined to 63 and $33 \%$ on days 4 and 6 , respectively. This reduction in viral efficiency suggests that cells susceptible to Adv during early therapy-induced senescence become progressively resistant to Adv during late senescence. Altogether, the results in Figure 5 suggest that a transition occurs during therapy-induced senescence, in which arrested senescent cells are converted from a permissive state of senescence escape and cell cycle re-entry to a restricted state with reinforced cell cycle arrest. Escape from senescence therefore occurs predominately during the earlier state as defined by CAR expression and adenoviral susceptibility.
We also examined the possibility that CAR expression, and therefore, adenoviral susceptibility may preferentially select a subpopulation of tumor cells that are resistant to chemotherapy. To address this possibility, we studied five consecutive escape colonies derived from sorted, marked senescent cells and found that all five colonies exhibited similar senescence responses and SA- $\beta$-gal expression (Supplementary Figure). These senescent escape colonies were previously shown to be equally sensitive to chemotherapy as compared with the parental line. ${ }^{19}$

\section{Magnitude of irreversibility conversion determines the} frequency of senescence escape. We postulated that the magnitude of surface CAR reduction or adenoviral marking could serve as a potential biomarker for therapy-induced senescence. This hypothesis was examined in vitro by determining whether the fraction of Adv-RFP marking on day 4 could be modulated by the initial dose of chemotherapy and whether this effect correlated with escape colony formation (Supplementary Figure 5). H1299 tumor cells were treated with increasing concentrations of CPT (15-150 nM) for 3 days, recovered in fresh media and infected with Adv-RFP on day 4. The relationship between colony formation and adenoviral marking was examined using a linear regression analysis, resulting in an $r^{2}$ coefficient of 0.918 (Figure 6). This finding provides evidence that adenoviral marking, and likely CAR expression, could serve as a surrogate tumor biomarker to predict the magnitude and 

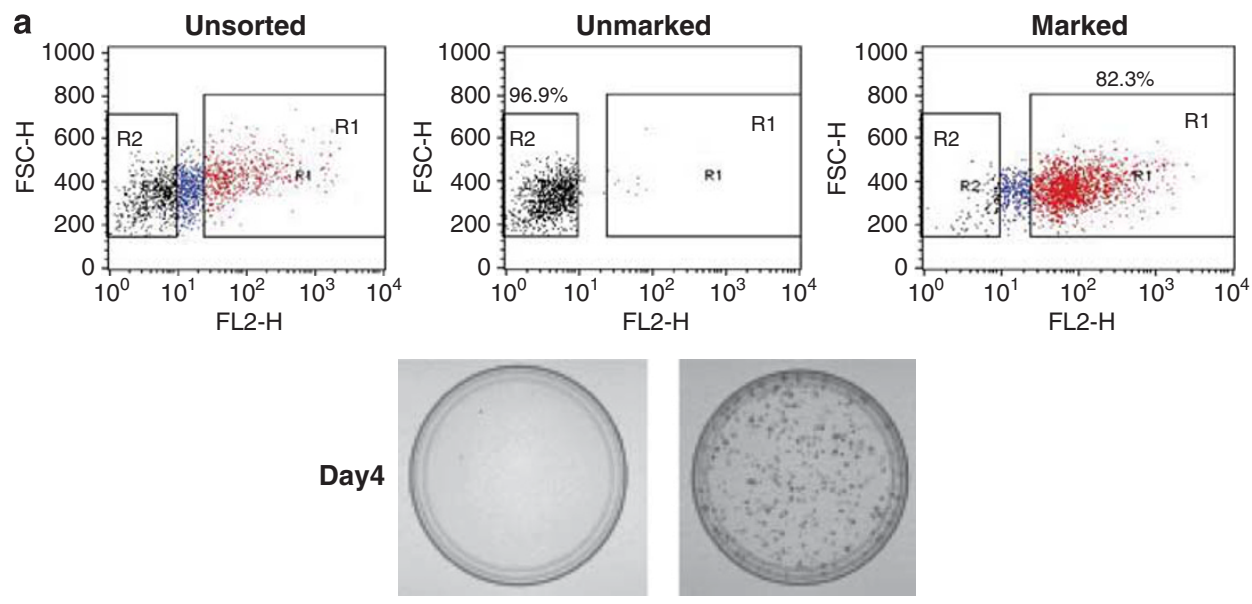

Colony Formation (per $5 \times 10^{4}$ cells)

\begin{tabular}{|c|c|c|}
\hline Day & Unmarked & Marked \\
\hline 2 & $2 \pm 1$ & $157 \pm 10$ \\
\hline 4 & 0 & $121 \pm 13$ \\
\hline 6 & $15 \pm 11$ & $234 \pm 15$ \\
\hline
\end{tabular}

b

\begin{tabular}{|c|c|c|}
\hline \multirow{2}{*}{$\begin{array}{c}\text { Date of Adv-LacZ } \\
\text { Marking }\end{array}$} & \multicolumn{2}{|c|}{$\%$ Colonies Marked } \\
\cline { 2 - 3 } & Expected & Results \\
\hline $\mathbf{0}$ & $89 \%$ & $100 \%(25 / 25)$ \\
\hline $\mathbf{4}$ & $42 \%$ & $100 \%(25 / 25)$ \\
\hline $\mathbf{6}$ & $12 \%$ & $100 \%(25 / 25)$ \\
\hline
\end{tabular}

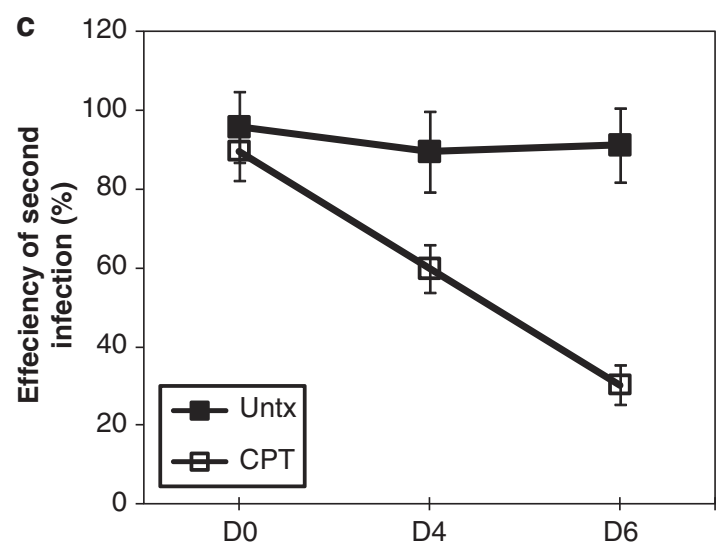

Figure 5 Relationship between marked and unmarked senescent cells. (a) Colony formation assays were performed on sorted H1299 cells following release from CPT. Sorting was performed according to the gates as indicated in the top panel. Equal numbers of marked and unmarked cells $\left(5 \times 10^{4}\right)$ were plated into fresh media and allowed to form colonies, stained at indicated days and colonies counted with results reported as mean \pm S.D. from three independent experiments $(N=3)$. (b) Senescent $\mathrm{H} 1299$ cells were infected with marker Adv containing the bacterial LacZ transgene at indicated days following CPT in the colony formation assay. Colonies were assayed for in situ $\beta$-gal expression (pH 7.0). The expected fraction of $\beta$-gal-expressing colonies from $\mathrm{H} 1299$ is based on mean results shown in Figure $2 \mathrm{~b}$. (c) Successive infections with markers Adv-RFP (day 0) and Adv-GFP (days 4 and 6) were performed on untreated and CPT-treated cells. Efficiency of secondary infection is determined by the number of dual fluorescent cells with respect to the total number of RFP-expressing cells measured by FACS. Error bars represent standard deviation of three independent experiments $(N=3)$

durability of senescence response during chemotherapy treatment.

Decline in adenoviral marking is influenced by $p 53$ status in cancer cell lines. To assess whether the decline in adenoviral marking observed during therapy-induced senescence is tumor-type specific or influenced by p53 status, we characterized the senescence response in 13 independent tumor lines, seven of which are wild type for the p53 gene (Table 1). Decline in adenoviral marking is defined as a consecutive reduction of marker Adv infectivity 3 and 6 days following chemotherapy. We found that nine out 
of 13 cancer cell lines exhibited decline of adenoviral marking during senescence, including six out of seven p53-wild-type tumor lines and three out of six p53-mutated or inactivated lines. Two p53-mutated tumor cell lines (CAMA-1 and HT29) showed insignificant baseline adenoviral susceptibility. All tested cell lines displayed evidence of senescence response based on their expression of SA- $\beta$-gal. In summary, cell lines with the highest SA- $\beta$-gal expression were found to be associated with the greatest baseline adenoviral marking. Our limited data suggest that both adenoviral marking and CAR downregulation may be more common in a p53-wildtype background.

CAR expression is downregulated in human rectal cancers treated with neoadjuvant chemoradiotherapy. Tumors resected from patients diagnosed with colorectal cancer were studied for CAR expression using anti-CAR antibody and FITC immunostaining (Figure 7). Tumor and adjacent normal tissues samples were collected from patients who underwent either chemoradiotherapy

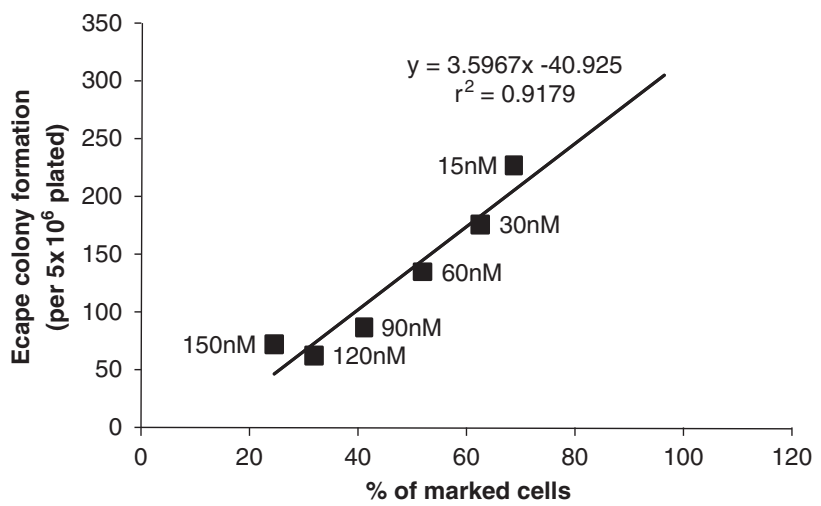

Figure 6 Linear correlation of adenoviral marking and colony formation based on dose effect: $\mathrm{H} 1299$ cells were treated with increasing CPT doses to generate a senescent pool for colony formation assay and simultaneously infected with Adv-RFP and analyzed by FACS for marker expression. Mean values from three experiments were plotted and linear regression analysis was performed with an $r^{2}$ correlation of 0.918 before surgery (lower panels) or surgery alone (upper panels). The freshly frozen tissues were examined for CAR expression. We found significantly less visible CAR staining in tumors resected from two patients treated with preoperative chemoradiotherapy compared with tumor samples from two patients who underwent surgery alone. Furthermore, CAR expression appeared unchanged in the normal colorectal mucosal tissue in all four patients regardless of previous chemotherapy treatment, thereby suggesting that differential CAR expression and staining patterns are tumor specific. To determine whether this is a CAR-specific treatment effect, we used a common pathological marker for epithelial neoplasms, cytokeratin 20 (CK20) and performed immunostaining of rectal tumor biopsies from patient $C$ (Figure 7) obtained before and after chemoradiation and observed no difference in CK20 expression (Supplementary Figure 7). ${ }^{32}$ This provocative finding in colorectal cancer patients suggests that CAR expression should be explored as an in vivo therapy-induced senescence biomarker in gastrointestinal cancer.

\section{Discussion}

In vivo cancer therapy response has been described as a heterogeneous mixture of apoptosis, mitotic catastrophe, autophagy, necrosis and therapy-induced senescence. ${ }^{33}$ Among these, therapy-induced senescence, characterized by prolonged cellular arrest, provides the most plausible explanation for the modest radiographic response typically observed in patients with solid tumors receiving chemotherapy. ${ }^{1,34}$ Non-operable solid tumors predictably recur in nearly all patients regardless of the extent of chemotherapy response, and this fact can be further explained by the ability of some therapy-induced senescent cells to circumvent and escape the terminal arrest program, resulting in tumor proliferation. In this study, we show that senescent cells in response to chemotherapy treatment can exist in two distinct transitional states functionally differentiated by their ability to re-enter the cell cycle. These states can be distinguished by their susceptibility to Adv transduction and characterized by the levels of surface CAR expression. The transition from a

Table 1 Characterization of adenoviral marking and senescence response in tumor cell lines

\begin{tabular}{|c|c|c|c|c|c|c|}
\hline \multirow[b]{2}{*}{ Cell line } & \multirow[b]{2}{*}{ Tumor origin } & \multirow[b]{2}{*}{ p53 status } & \multirow{2}{*}{$\begin{array}{l}\text { Chemotherapy } \\
\text { dose }\end{array}$} & \multirow{2}{*}{$\begin{array}{c}\text { SA- } \beta \text {-gal } \\
\text { expression }\end{array}$} & \multicolumn{2}{|c|}{ Adv marking $(\%)^{a}$} \\
\hline & & & & & Baseline & Decline (6 day) \\
\hline$A 549$ & Lung & wt & СРT $100 \mathrm{nM}$ & ++++ & 87 & $Y$ \\
\hline H1299 & Lung & null & СРT $60 \mathrm{nM}$ & ++++ & 92 & $\mathrm{Y}$ \\
\hline MCF7 & Breast & wt & Dox $25 \mathrm{nM}$ & ++ & 26 & $\mathrm{Y}$ \\
\hline CAMA-1 & Breast & mut & CPT $250 \mathrm{nM}$ & + & $<2$ & $N A^{b}$ \\
\hline$B \times P C-3$ & Pancreas & wt & CPT $200 \mathrm{nM}$ & ++ & 68 & $\mathrm{Y}$ \\
\hline PANC-1 & Pancreas & mut & СРТ $120 \mathrm{nM}$ & ++ & 75 & $\mathrm{Y}$ \\
\hline HCT 116 & Colorectal & wt & СРТ $40 \mathrm{nM}$ & ++++ & 78 & $\mathrm{Y}$ \\
\hline LoVo & Colorectal & wt & СРТ $1 \mathrm{nM}$ & + & 20 & $\mathrm{Y}$ \\
\hline SW48 & Colorectal & wt & СРT $10 \mathrm{nM}$ & ++ & 23 & $\mathrm{~N}$ \\
\hline LS174T & Colorectal & wt & Oxali $1 \mu \mathrm{M}$ & +++ & 15 & $\mathrm{Y}$ \\
\hline$F E T$ & Colorectal & mut & CPT $400 \mathrm{nM}$ & + & 10 & $\mathrm{~N}$ \\
\hline HT29 & Colorectal & mut & СРТ $10 \mathrm{nM}$ & + & 6 & $N A^{b}$ \\
\hline Hela & Cervical & Inactivated & Dox $600 \mathrm{nM}$ & +++ & 69 & $\mathrm{Y}$ \\
\hline
\end{tabular}

Abbreviations: wt, wild type; mut, mutant; CPT, camptothecin; Dox, doxorubicin; Oxali, oxaliplatin; NA, not applicable. ${ }^{\mathrm{a}}$ Adenoviral marking determined by FACS (described in Figure 2). ' Untreated baseline marking $<10 \%$ considered insufficient to assess decline (all FACS experiments associated with $10 \%$ variability) 


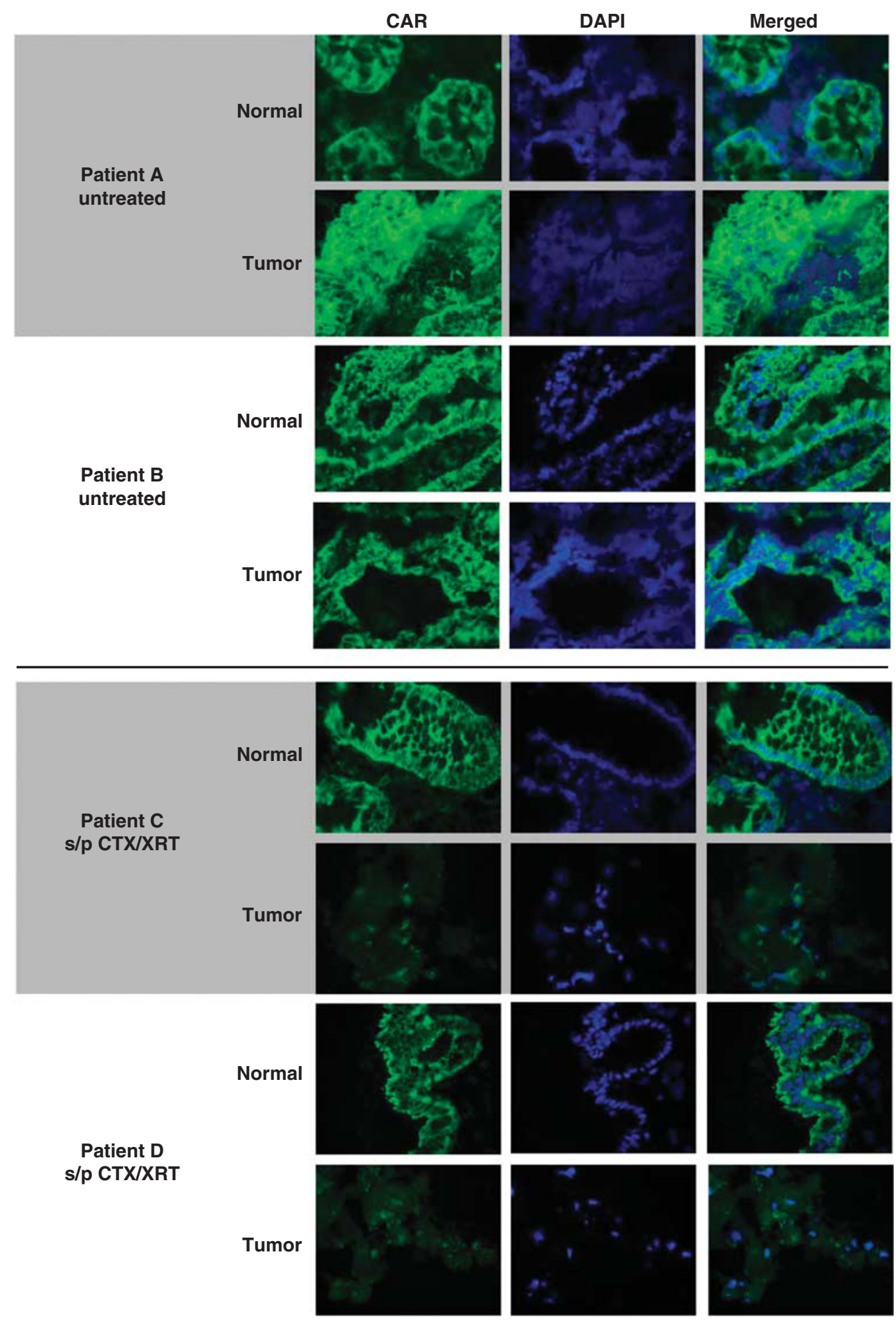

Figure 7 CAR expression in colorectal adenocarcinoma following chemoradiotherapy: human tumor and adjacent normal tissue specimens were obtained from colorectal cancer patients undergoing curative resection treated with or without preoperative chemotherapy and radiation (CTX/XRT). Freshly frozen specimens were stained with CAR primary antibody (Santa Cruz) followed with FITC-conjugated secondary antibody or with DAPI. Immunofluorescence micrographs $(\times 40)$ of CAR, DAPI and merged images are shown

'reversible' to an 'irreversible' state therefore represents a significant biological barrier within senescence. Overcoming this barrier commits senescent cells to terminal arrest and eventual cell death. A small subpopulation of senescent cancer cells fails to undergo this transition and thus retains the capacity to re-enter the cell cycle leading to tumor progression. It is likely that the magnitude of the biological transition to irreversibility represents a functional determinant of treatment efficacy. Such a senescence barrier has been previously described in SVts8 fibroblast cells where the $\mathrm{p} 16^{\mathrm{INK} 4 \mathrm{a}} / \mathrm{Rb}$ 
pathway appears to cooperate with mitogenic signals to enforce an irreversible block of cytokinesis through the production of reactive oxygen species. ${ }^{35}$ The biological barrier in $\mathrm{H} 1299$ cancer cells is presently unknown as $p 16^{\mathrm{INK} 4 a}$ is silenced in these cells. ${ }^{19}$

The efficacy of increasing drug dose in chemotherapy forms the theoretical basis for dose intensity in cancer therapeutics. ${ }^{36}$ However, dose escalation is limited by normal tissue tolerance as well as the failure of dose escalation to eradicate all disease. Indeed, clinical trials utilizing high-dose chemotherapy for solid tumors have been largely ineffective with limited clinical benefit. ${ }^{36}$ In this study, we show that escalating CPT dose reduces both the population of reversibly arrested senescent cells and the formation of escape colonies. This observation suggests that the magnitude and rate of conversion to irreversible therapy-induced senescence can be modulated by dose intensity and quantified by CAR expression; however, even a 10-fold increase of chemotherapy cannot overcome the ability of some senescent cells to circumvent conversion to irreversible arrest. Taken together this suggests a potential role for biomarkers to assess in vivo senescence response. An appealing candidate marker is CAR, which is ubiquitously expressed on the surface of most epithelial tumors, including prostate, endometrial and other common gastrointestinal malignancies. ${ }^{25,37,38}$ The level of CAR expression, however, appears to vary widely depending on tissue type and histological grade of the tumor. The demonstration of surface CAR downregulation in human tumor specimens following preoperative chemotherapy treatment closely parallels expression profiles characterized in tissue culture models. Thus, chemotherapeutic modulation of CAR expression could be studied as a surrogate biomarker for senescence response.

We also propose that CAR expression can be used as a novel methodology for the isolation of senescent cell subpopulations with characteristic biological states based on their ability to re-enter the cell cycle and likely represent cells in either reversible or irreversible transition states. The approach outlined here should enable comparative studies of early molecular events that regulate senescence reversibility. Understanding these cellular events may unveil mechanisms by which cancers can bypass this critical transition in therapyinduced senescence. One such example is the role of telomere disruption and telomerase regulation in senescent tumor cells. We have found that therapy-induced senescent cells are frequently accompanied by significant telomere loss. In preliminary experiments, we have observed that senescent cells, which are able to escape arrest and regain proliferative capacity, partially recover the lost telomere. These preliminary results suggest that telomere disruption and recovery may be an underlying mechanism of the irreversibility barrier. This hypothesis is supported by the finding of significantly higher telomerase activity in Adv marked senescent cells compared with unmarked cells (Supplementary Figure 6). Our report illustrates the utility of CAR expression and adenoviral marker transduction as convenient methodologies to study fundamental regulatory mechanisms in therapyinduced senescence. Successful application of this approach to characterize transitional states in cancer senescence may lead to the discovery of potential drug targets and novel therapeutic strategies to improve cancer clinical outcomes.

\section{Materials and Methods}

Cell culture. NCl-H1299 (H1299) lung carcinoma (ATCC, Manassas, VA, USA) and BxPC-3 pancreatic adenocarcinoma (ATCC) cells were cultured in RPMI 1640 supplemented with $10 \%$ fetal bovine serum and $1 \%$ penicillin/streptomycin (BioWhittaker, Walkersville, MD, USA). HCT 116 colon carcinoma cells (ATCC) were cultured in McCoy's 5a medium supplemented with $10 \%$ fetal bovine serum and $1 \%$ penicillin/streptomycin. Cells were treated with chemotherapy at $15 \%$ to $30 \%$ cell density for 1-3 days, rinsed with PBS and allowed to recover at indicated times in complete growth medium for specific assays. $\mathrm{H} 1299$ and BxPC-3 cells were treated with CPT and HCT 116 was treated with doxorubicin at indicated concentrations and recovered as shown in the schema (Figure 1a). G1 arrest was induced in $\mathrm{H} 1299$ cells by serum starvation in RPMI with $0.5 \%$ FBS for $72 \mathrm{~h}$, whereas G2/M arrest was induced by treating $\mathrm{H} 1299$ cells with $20 \mathrm{~nm}$ nocodazole for 16-24 h (Sigma-Aldrich, St Louis, MO, USA). The cancer cell lines including LoVo, SW48, LS174T and FET were provided as a kind gift from Dr. William Grady (Fred Hutchinson Cancer Research Center, Seattle, WA, USA) and HT29 was a generous gift from Dr. Steven Libutti (NCI/NIH, Bethesda, MD, USA). The remaining cell lines including A549, MCF7, CAMA-1, PANC-1 and HeLa were obtained from ATCC.

Construction and expression of marker Adv. RNAi-ready pSirenDNR-DsRed-Express vector was obtained from Clontech (Mountain View, CA, USA), which contains the RFP. For our purpose, we annealed the linearized vector by T4 DNA ligase without an insert and transferred the RFP fluorescent marker to adenoviral X-acceptor vector by cre recombinase according to the manufacturer's protocol (AdenoX kit; Clontech). The recombinant viral vector was screened and amplified in Escherichia coli, packaged and amplified in HEK 293 as per the manufacturer's instruction. Adv containing the LacZ gene was constructed as per the manufacturer's instructions (AdenoX kit; Clontech) and Adv containing enhanced GFP was a kind gift from Dr. Liu Yang (University of Washington, Seattle, WA, USA).

Immunofluorescence microscopy. Adv-RFP, Adv-GFP and CAR expression were detected by fluorescence microscopy (Nikon eclipse E-800). For Adv-RFP or Adv-GFP expression, tumor cells were incubated in multi-chamber slides with marker Adv for $4 \mathrm{~h}$, washed in PBS and replaced in respective complete growth medium. After overnight incubation, cells were washed and fixed in $1 \%$ paraformaldehyde. The cells were then permeabilized with $0.2 \%$ Triton X-100 and stained with $0.2 \% \mathrm{DAPI}$ solution and examined under the fluorescence microscope. For CAR expression, cells were incubated in multi-chamber slides with anti-CAR antibody at 1:50 dilution in 1\% BSA/PBS (Abcam, Cambridge, MA, USA) for $1 \mathrm{~h}$ and further incubated with FITC-conjugated anti-mouse secondary antibody (1:200). After fixation in 1\% paraformaldehyde, the cells were examined using fluorescence microscopy. Tumor and normal tissue samples were collected from colorectal cancer patients undergoing elective resection following standard informed consent and protocols approved by the VA Puget Sound Human Subjects Institutional Research Board (IRB No. 01468, Colorectal Cancer Treatment and Cellular Senescence). Tumor and tissue samples were freshly frozen in Tissue-Tek OCT (Sakura Finetek, Alphen aan den, the Netherlands), cryosectioned and immunostained with anti-CAR (Santa Cruz Biotechnology, Santa Cruz, CA, USA) and FITC-conjugated secondary antibody as described above.

FACS analysis and cell sorting. RFP-, GFP- and CAR-expressing cells were analyzed and sorted by FACS (Becton-Dickinson FACS caliber). Tumor cells used in adenoviral marking experiments were incubated with Adv for $4 \mathrm{~h}$, washed with PBS and recovered in complete growth medium. After overnight recovery, the cells were collected, fixed in $1 \%$ paraformaldehyde and analyzed by flow cytometry. For CAR expression analysis, the cells were collected and incubated with 1:50 mouse anti-CAR antibody (Abcam) in 1\% BSA/PBS for $1 \mathrm{~h}$ and with 1:200 secondary FITC anti-mouse antibody for $30 \mathrm{~min}$ without fixation. The expression of CAR was detected by FACS analysis. In the FACS sorting experiments, tumor cells were treated as previously described following Adv infection or CAR immunostaining, sorted and collected in $50 \mathrm{ml}$ Falcon tubes pre-coated with $4 \%$ BSA/PBS. Cell suspensions were concentrated by centrifugation at 1500 r.p.m. for $5 \mathrm{~min}$ and resuspended in complete growth medium for further analysis. 
MTT cell proliferation assay. Treated $\mathrm{H} 1299$ tumor cells were infected with Adv-RFP and FACS sorted into 'marked' and 'unmarked' cell populations. MTT cell proliferation assays were performed on serial dilutions of these cell populations ranging from $1 \times 10^{3}$ to $1 \times 10^{6}$ cells according to the manufacturer's recommendations (ATCC). Absorbance at $570 \mathrm{~nm}$ was measured from triplicate plates using a microtiter plate reader.

Colony formation assay and staining. The cells were infected with Adv-RFP on day 4 following chemotherapy and sorted according to their RFP expression. Indicated numbers of sorted cells were replated in complete growth medium and allowed to form colonies typically observed after $10-17$ days. The plate colonies were then fixed in $1 \%$ paraformaldehyde, stained with $0.2 \%$ trypan blue, scanned and counted using an Alphalmager system (Cell Biosciences, Santa Clara, CA, USA). In situ X-gal assay was performed at pH 7.0 as per the manufacturer's recommendation (Promega, Madison, WI, USA). SA- $\beta$-gal in situ staining was performed as we have reported previously. ${ }^{19}$

Image processing and reproduction. Images of gel and digital photographs were generated with Adobe Photoshop CS4 and Illustrator CS4.

Statistical analysis. Statistical analysis was performed as specified using Microsoft Office 2007 package.

\section{Conflict of interest}

The authors declare no conflict of interest.

Acknowledgements. This material is based on work supported by the Veteran Affairs Office of Research \& Development Medical Research Service (Biomedical Laboratory R\&D Career Development Award-2 to PCW) and National Cancer Institute (R01-CA113892-01 to DYW). We thank Dr. Liu Yang for his critical reading of our manuscript and his insightful suggestions.

1. Chang BD, Xuan Y, Broude EV, Zhu H, Schott B, Fang J et al. Role of p53 and p21waf1/ cip1 in senescence-like terminal proliferation arrest induced in human tumor cells by chemotherapeutic drugs. Oncogene 1999; 18: 4808-4818.

2. Hwang ES. Replicative senescence and senescence-like state induced in cancer-derived cells. Mech Ageing Dev 2002; 123: 1681-1694.

3. Mooi WJ, Peeper DS. Oncogene-induced cell senescence - halting on the road to cancer. N Engl J Med 2006; 355: 1037-1046.

4. Serrano M, Lin AW, McCurrach ME, Beach D, Lowe SW. Oncogenic ras provokes premature cell senescence associated with accumulation of p53 and p16INK4a. Cell 1997; 88: 593-602.

5. Feagins LA, Souza RF. Molecular targets for treatment of Barrett's esophagus. Dis Esophagus 2005; 18: 75-86.

6. Greenberg RA. Telomeres, crisis and cancer. Curr Mol Med 2005; 5: 213-218.

7. Ha L, Merlino G, Sviderskaya EV. Melanomagenesis: overcoming the barrier of melanocyte senescence. Cell Cycle 2008; 7: 1944-1948.

8. Chen Z, Trotman LC, Shaffer D, Lin HK, Dotan ZA, Niki M et al. Crucial role of p53-dependent cellular senescence in suppression of Pten-deficient tumorigenesis. Nature 2005; 436: 725-730.

9. Dirac AM, Bernards R. Reversal of senescence in mouse fibroblasts through lentiviral suppression of p53. J Biol Chem 2003; 278: 11731-11734.

10. Sage J, Miller AL, Perez-Mancera PA, Wysocki JM, Jacks T. Acute mutation of retinoblastoma gene function is sufficient for cell cycle re-entry. Nature 2003; 424: 223-228.

11. Dai CY, Enders GH. p16 INK4a can initiate an autonomous senescence program. Oncogene 2000; 19: 1613-1622.

12. Jarrard DF, Sarkar S, Shi Y, Yeager TR, Magrane G, Kinoshita H et al. p16/pRb pathway alterations are required for bypassing senescence in human prostate epithelial cells. Cancer Res 1999; 59: 2957-2964.

13. Christov KT, Shilkaitis AL, Kim ES, Steele VE, Lubet RA. Chemopreventive agents induce a senescence-like phenotype in rat mammary tumours. Eur J Cancer 2003; 39: 230-239.

14. Elmore LW, Rehder CW, Di X, McChesney PA, Jackson-Cook CK, Gewirtz DA et al. Adriamycin-induced senescence in breast tumor cells involves functional p53 and telomere dysfunction. J Biol Chem 2002; 277: 35509-35515.

15. Groth A, Weber JD, Willumsen BM, Sherr CJ, Roussel MF. Oncogenic Ras induces p19ARF and growth arrest in mouse embryo fibroblasts lacking p21Cip1 and p27Kip1 without activating cyclin D-dependent kinases. J Biol Chem 2000; 275: 27473-27480.

16. Roninson IB. Tumor cell senescence in cancer treatment. Cancer Res 2003; 63 2705-2715.

17. Fang L, Igarashi M, Leung J, Sugrue MM, Lee SW, Aaronson SA. p21Waf1/Cip1/Sdi1 induces permanent growth arrest with markers of replicative senescence in human tumor cells lacking functional p53. Oncogene 1999; 18: 2789-2797.

18. Wang Y, Blandino G, Givol D. Induced p21 waf expression in $\mathrm{H} 1299$ cell line promotes cell senescence and protects against cytotoxic effect of radiation and doxorubicin. Oncogene 1999; 18: 2643-2649.

19. Roberson RS, Kussick SJ, Vallieres E, Chen SY, Wu DY. Escape from therapy-induced accelerated cellular senescence in p53-null lung cancer cells and in human lung cancers. Cancer Res 2005; 65: 2795-2803.

20. Bergelson JM, Cunningham JA, Droguett G, Kurt-Jones EA, Krithivas A, Hong JS et al. Isolation of a common receptor for Coxsackie B viruses and adenoviruses 2 and 5 . Science 1997; 275: 1320-1323.

21. Honda T, Saitoh H, Masuko M, Katagiri-Abe T, Tominaga K, Kozakai I et al. The coxsackievirus-adenovirus receptor protein as a cell adhesion molecule in the developing mouse brain. Brain Res Mol Brain Res 2000; 77: 19-28.

22. Roelvink PW, Mi Lee G, Einfeld DA, Kovesdi I, Wickham TJ. Identification of a conserved receptor-binding site on the fiber proteins of CAR-recognizing adenoviridae. Science 1999; 286: 1568-1571.

23. Tomko RP, Johansson CB, Totrov M, Abagyan R, Frisen J, Philipson L. Expression of the adenovirus receptor and its interaction with the fiber knob. Exp Cell Res 2000; 255: 47-55.

24. Stecker K, Koschel A, Wiedenmann B, Anders M. Loss of Coxsackie and adenovirus receptor downregulates alpha-catenin expression. $\mathrm{Br} J$ Cancer 2009; 101: 1574-1579.

25. Anders M, Rosch T, Kuster K, Becker I, Hofler H, Stein HJ et al. Expression and function of the coxsackie and adenovirus receptor in Barrett's esophagus and associated neoplasia. Cancer Gene Ther 2009; 16: 508-515.

26. Communal C, Huq F, Lebeche D, Mestel C, Gwathmey JK, Hajjar RJ. Decreased efficiency of adenovirus-mediated gene transfer in aging cardiomyocytes. Circulation 2003; 107: 1170-1175.

27. Hung SC, Lu CY, Shyue SK, Liu HC, Ho LL. Lineage differentiation-associated loss of adenoviral susceptibility and Coxsackie-adenovirus receptor expression in human mesenchymal stem cells. Stem Cells 2004; 22: 1321-1329.

28. Coyne CB, Bergelson JM. CAR: a virus receptor within the tight junction. Adv Drug Deliv Rev 2005; 57: 869-882.

29. Hemmi S, Geertsen R, Mezzacasa A, Peter I, Dummer R. The presence of human coxsackievirus and adenovirus receptor is associated with efficient adenovirus-mediated transgene expression in human melanoma cell cultures. Hum Gene Ther 1998; 9: 2363-2373.

30. Kim M, Sumerel LA, Belousova N, Lyons GR, Carey DE, Krasnykh V et al. The coxsackievirus and adenovirus receptor acts as a tumour suppressor in malignant glioma cells. Br J Cancer 2003; 88: 1411-1416.

31. Pearson AS, Koch PE, Atkinson N, Xiong M, Finberg RW, Roth JA et al. Factors limiting adenovirus-mediated gene transfer into human lung and pancreatic cancer cell lines. Clin Cancer Res 1999; 5: 4208-4213.

32. Chu P, Wu E, Weiss LM. Cytokeratin 7 and cytokeratin 20 expression in epithelial neoplasms: a survey of 435 cases. Mod Pathol 2000; 13: 962-972.

33. Portugal J, Bataller M, Mansilla S. Cell death pathways in response to antitumor therapy. Tumori 2009; 95: 409-421.

34. Schmitt CA, Fridman JS, Yang M, Lee S, Baranov E, Hoffman RM et al. A senescence program controlled by p53 and p16INK4a contributes to the outcome of cancer therapy. Cell 2002; 109: 335-346.

35. Takahashi A, Ohtani N, Yamakoshi K, lida S, Tahara H, Nakayama K et al. Mitogenic signalling and the p16INK4a-Rb pathway cooperate to enforce irreversible cellular senescence. Nat Cell Biol 2006; 8: 1291-1297.

36. Pedrazzoli P, Rosti G, Secondino S, Carminati O, Demirer T. High-dose chemotherapy with autologous hematopoietic stem cell support for solid tumors in adults. Semin Hematol 2007; 44: 286-295.

37. Giaginis CT, Zarros AC, Papaefthymiou MA, Papadopouli AE, Sfiniadakis IK, Theocharis SE. Coxsackievirus and adenovirus receptor expression in human endometrial adenocarcinoma: possible clinical implications. World J Surg Oncol 2008; 6: 59.

38. Rauen KA, Sudilovsky D, Le JL, Chew KL, Hann B, Weinberg V et al. Expression of the coxsackie adenovirus receptor in normal prostate and in primary and metastatic prostate carcinoma: potential relevance to gene therapy. Cancer Res 2002; 62: 3812-3818.

(c)

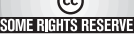

Cell Death and Disease is an open-access journal published by Nature Publishing Group. This work is licensed under the Creative Commons Attribution-Noncommercial-No Derivative Works 3.0 Unported License. To view a copy of this license, visit http://creativecommons.org/licenses/by-nc-nd/3.0/

\section{Supplementary Information accompanies the paper on Cell Death and Disease website (http://www.nature.com/cddis)}

